\title{
Infrared Emission from Supernova Remnants: Formation and Destruction of Dust
}

\author{
Brian J. Williams and Tea Temim
}

\begin{abstract}
We review the observations of dust emission in supernova remnants (SNRs) and supernovae ( $\mathrm{SNe}$ ). Theoretical calculations suggest that $\mathrm{SNe}$, particularly core-collapse, should make significant quantities of dust, perhaps as much as a solar mass. Observations of extragalactic $\mathrm{SNe}$ have yet to find anywhere near this amount, but this may be the result of observational limitations. SN 1987A, in the process of transitioning from a SN to an SNR, does show signs of a significant amount of dust forming in its ejecta, but whether this dust will survive the passage of the reverse shock to be injected into the ISM is unknown. IR observations of SNRs have not turned up significant quantities of dust, and the dust that is observed is generally swept-up by the forward shock, rather than created in the ejecta. Because the shock waves also destroy dust in the ISM, we explore the question of whether $\mathrm{SNe}$ might be net destroyers, rather than net creators of dust in the universe.
\end{abstract}

\section{Introduction}

Supernova remnants (SNRs) provide a laboratory for the study of various aspects of the evolution of the interstellar medium (ISM) across the electromagnetic spectrum. SNRs are often bright in the infrared (IR), where both continuum emission from warm dust and line emission from molecules and low ionization atomic states of various elements are observed. The ejecta from SNRs enrich the ISM of the Galaxy with metal-rich products such as silicon, iron, oxygen, and magnesium. These are

Brian J. Williams

CRESST/USRA and X-ray Astrophysics Laboratory, NASA/GSFC, Code 662, Greenbelt, MD, USA, e-mail:brian.j.williams@nasa.gov

Tea Temim

CRESST/UMCP and Observational Cosmology Laboratory, NASA/GSFC, Code 665, Greenbelt, MD, USA e-mail: tea.temimenasa.gov 
the same elements that are predominantly found in dust grains, leading to the conclusion that grains might be formed within the expanding, cooling ejecta.

Dust plays an important role in all stages of galaxy evolution, as the life cycle of dust grains and the amount and relative abundances present in the ISM are determined by the balance between grain formation, modification, and destruction. Dust heated in the powerful shock waves of SNRs radiates strongly at IR wavelengths, so IR observations provide a means to study both the properties of ISM dust and the formation and destruction mechanisms in ways that are not otherwise feasible. The conclusion that $\mathrm{SNe}$ are responsible for creating most of the dust in the universe is not a closed case. During the remnant phase, the shock wave races out into the ISM, heating and compressing the interstellar or circumstellar gas. This gas, heated to an X-ray emitting plasma of several million degrees, collides with dust grains embedded within the medium, heating them to temperatures of up to a few hundred degrees, but also modifying and destroying them in the process. Even the newly formed grains within the ejecta are subject to destruction by the reverse shock wave, and it may be that $\mathrm{SNe}$, on the whole, destroy more dust than they create.

In this work, we review some of the basic theoretical and observational work behind IR emission in SNe and SNRs. We discuss the heating and cooling mechanisms for gas and dust in remnants, and discuss dust formation in SN ejecta. In general, the amount of dust detected during the SN phase is quite small, only a few thousandths of a solar mass. Most SNe fade from view in the IR within a few months, though some, like Type IIn SNe, remain bright for years post-explosion, presumably due to a significant amount of dust created in the pre-SN outflows. Young SNRs (where young is loosely defined as a few hundred to a few thousand years old) are typically bright mid-IR sources, and Spitzer has returned many spectacular images and spectra of these remnants. The shape of the dust spectrum can serve as a diagnostic of both the grain properties, and the hot, X-ray emitting plasma. We also discuss the destruction of dust by the forward and reverse shock waves.

We summarize recent progress made in these areas, which has grown by leaps and bounds over the past decade with the remarkable success of the Spitzer and Herschel missions. The focus is on Galactic and Magellanic Clouds (MCs) observations of SNRs, because the resolved nature of remnants at these distances allows for detailed study of the interaction of the shock wave with dust in the ambient Galactic medium. While core-collapse $\mathrm{SNe}$ do show some evidence for dust formation in their ejecta, Type Ia SNe do not. We also discuss extragalactic observations of $\mathrm{SNe}$, and finally, address the question of whether $\mathrm{SNe}$, as a whole, are net producers or destroyers of dust in the universe.

\section{IR Emission from SNRs}

SNRs have several emission mechanisms, both thermal and nonthermal, in the IR. Thermal emission can manifest as either a continuum from warm dust grains or as line emission from atomic or molecular gas. Rarely, nonthermal emission is present 
in the form of synchrotron emission from relativistic electrons accelerated by shock waves. These are not mutually exclusive, and some remnants have emission from all three. The dominant emission mechanism depends on several factors, but the primary determinant is the evolutionary stage of the remnant, which can be parameterized as the product of the remnant's age and the density of the material into which the forward shock wave is expanding.

In general, remnants at an advanced evolutionary stage (either because they are older or have encountered a denser medium) are dominated by line emission from atomic and molecular gas. The gas temperatures in these have cooled sufficiently to allow recombination to take place, and the atomic fine structure lines from common elements such as $\mathrm{Fe}, \mathrm{O}, \mathrm{Si}$, and $\mathrm{Ne}$ dominate the spectrum. In shocked molecular gas, $\mathrm{H}_{2}$ is the dominant coolant. We refer the reader to Reach et al. (2006) for a more thorough review of the IR line emission in advanced SNRs. These remnants are also bright in optical wavebands, showing up as radiative shocks.

In young remnants, on the other hand, shock waves are still mostly non-radiative, meaning that the plasma temperature in the post-shock environment is still too high (the thermal emission from this gas is in the X-ray band) for recombination to occur. As a result, these remnants emit very little in optical wavebands, but can emit significant IR emission as thermal continuum from dust grains, warmed by collisions with energetic particles in the post-shock gas (we detail the heating mechanism for this in Section 2.1. In some young remnants, this thermal dust continuum is the only source of emission in the IR. For examples of this, see Williams et al. (2011).

Most remnants show a mix of continuum and line emission sources, though the exact physical locations of their origins may be distinct. As an example of this, we show the integrated spectrum from Kepler's SNR in Figure 1. The spectrum contains strong continuum emission and several bright emission lines. Williams et al. (2012) interpreted the continuum as arising from the fast non-radiative shocks, while the line emission comes from the slower, radiative shocks, seen in the optical. Finally, some remnants, like Cas A, are bright enough that a faint contribution from synchrotron emission is detectable.

\subsection{Heating Mechanisms for Dust in SNe and SNRs}

Dust grains in the ISM are microscopic, ranging in size from a few nanometers to several microns, and are generally composed of refractory elements, such as carbon, magnesium, silicon, and iron. On average, about $0.1-1 \%$ of the mass of the ISM is contained in dust grains, with the remainder being in the gaseous phase (the bulk of that being hydrogen and helium). ISM grains have a steep distribution in size, roughly following a power-law given by $N(a) \propto a^{-3.5}$, where $a$ is the grain radius.

In general, dust grains are heated in one of two ways: by absorption of radiation or by direct collision with energetic particles. These two mechanisms are not mutually exclusive. Most dust heating in the universe is done via radiation. In the ambient ISM, grains are heated by the interstellar radiation field, primarily ultra- 


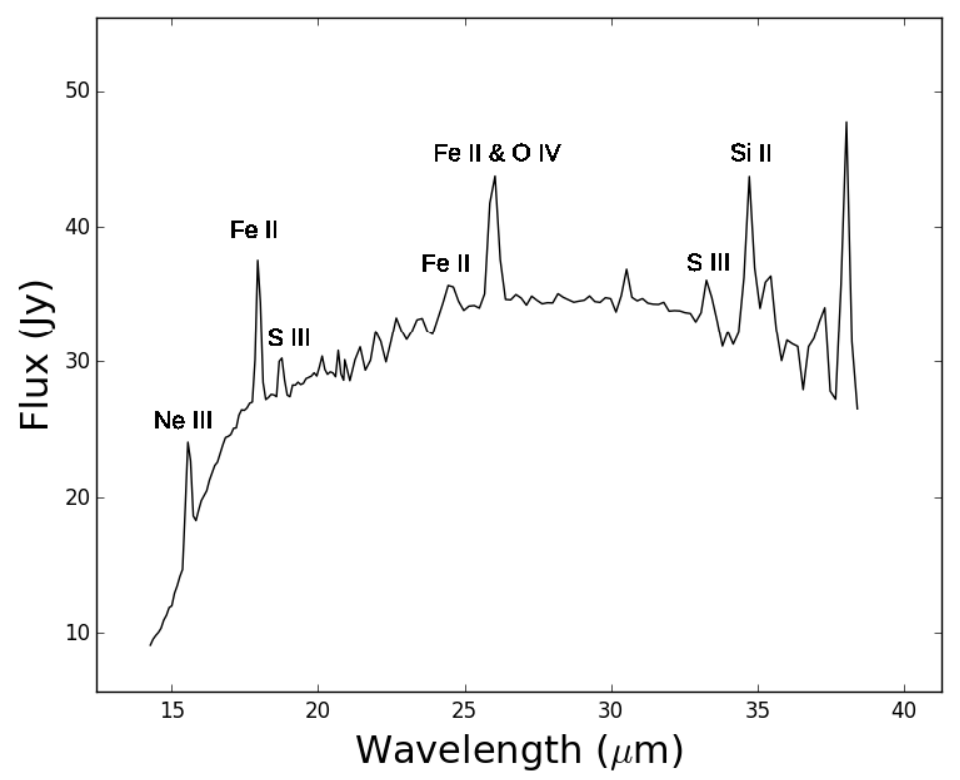

Fig. 1 Spitzer mid-IR spectrum of Kepler's SNR. The continuum results from warm dust at $\sim 100$ K. Several emission lines from low-ionization state ions are visible.

violet starlight, to temperatures of 10-20 K. Dust much closer to bright sources, such as stars, can be heated to significantly higher temperatures, and is often seen in mid-IR observations. Regardless of the heating mechanism, dust grains will cool via radiation in the IR as a modified blackbody, where the exact shape of the spectrum depends on the optical properties of the grains themselves.

In $\mathrm{SNe}$, there are several radiative mechanisms which can heat dust. The simplest mechanism is the extreme luminosity from the SN itself, powered by either radioactive decay or circumstellar interaction of the shock wave and the dense material it is plowing into. Grain temperatures observed in SNe can exceed $500 \mathrm{~K}$, so observations of dust in SNe must be done in the near-IR.

In young remnants, the bulk of the radiation emitted by the shocked plasma is at $\mathrm{X}$-ray energies. But since dust grains are not efficient absorbers of X-rays, the heating of grains in these remnants is dominated by collisions with hot electrons and ions (Draine \& Salpeter 1979), the same electrons and ions responsible for the Xray emission observed behind the forward shock in SNRs. This physical connection between the X-ray and IR emission mechanisms accounts for the similar morphologies of young remnants seen in these two wavebands (Williams et al. 2006). See Figure 2 for an example using the remnant of Kepler's SN of 1604 A.D.

In the late SNR phase, slow, radiative shocks $\left(<150 \mathrm{~km} \mathrm{~s}^{-1}\right)$ can emit significant amounts of optical/UV radiation due to the rapidly cooling gas in the post-shock environment. This radiation generally becomes the dominant heating source in older 
remnants, since young remnants are usually dominated by non-radiative shocks. The dust in older remnants tends to be cooler, generally in the $20-50 \mathrm{~K}$ range, emitting mostly in the far-IR. Virtually all of the emission from older remnants is dominated by swept-up ISM dust.
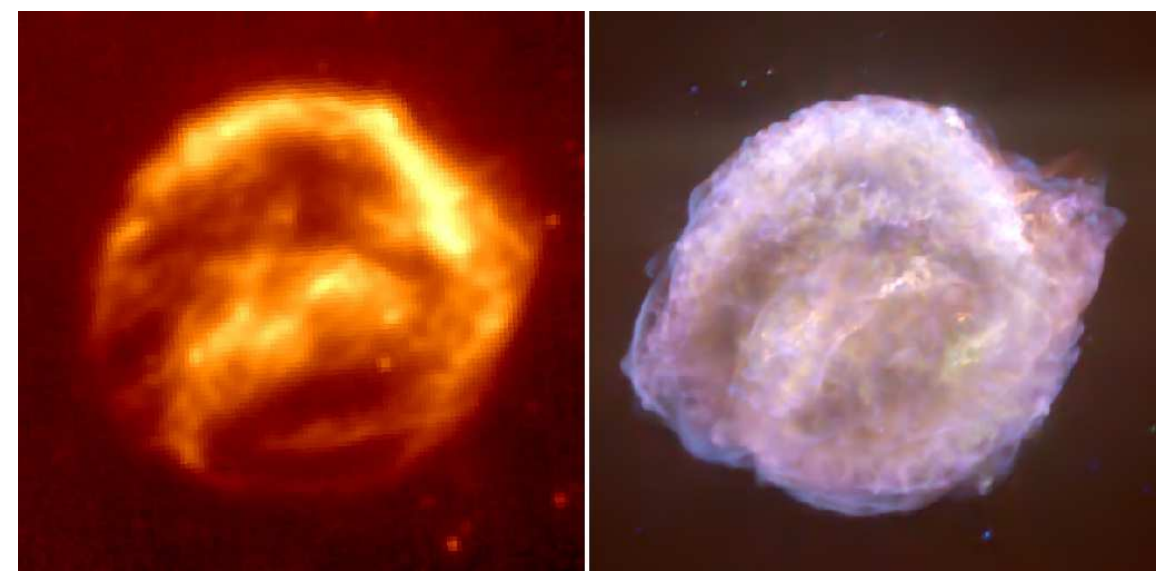

Fig. 2 Left: Spitzer $24 \mu \mathrm{m}$ image of Kepler's SNR (PI: W.P. Blair). Right: Chandra 3-color mosaic (PI: S.P. Reynolds), with 0.3-0.72 keV emission in red, 0.72-1.7 keV emission in green, and 1.7-7.0 $\mathrm{keV}$ emission in blue. The morphological connections between the two wavebands are obvious; see the text for details. The image is approximately $5^{\prime}$ on a side.

The heating rate for a grain immersed in a hot plasma is given by

$$
H=\left(\frac{32}{\pi m}\right)^{1 / 2} \pi a^{2} n(k T)^{3 / 2} h(a, T),
$$

where $m$ is the mass of the impinging particle (proton, electron, etc.), $a$ is the radius of the grain, $n$ is the density of the gas, $k T$ is the temperature of the gas, and $h(a, T)$ is a function that describes the efficiency of the energy deposition rate of a particle at a given $T$ for a grain with radius $a$. It can be immediately seen from this equation that at a given temperature, electrons will dominate the heating over protons, since their mass is much smaller and they move much faster. Young remnants, such as Kepler's SNR, have grain temperatures in the 50-200 K range, and emit most of their light between $20-50 \mu \mathrm{m}$. If the temperature of the plasma is known reasonably well from X-ray observations, the density of the post-shock gas can be used as a free parameter to match the IR spectral energy distribution of the dust. For instance, in Kepler's SNR, a combined IR/X-ray analysis showed that the density of the medium behind the forward shock is $\sim 50 \mathrm{~cm}^{-3}$, orders of magnitude above what one would expect for the "typical" ISM at Kepler's location in the Galaxy (Williams et al. 2012), further solidifying the case that Kepler is a Type Ia remnant with circumstellar interaction.

The radiative heating of grains by a nearby bright optical/UV source is given by 


$$
H=\frac{\pi a^{2} \int f(v, a) L_{v}(v) Q_{a b s}(v, a) d v}{4 \pi r^{2}},
$$

where $f(v, a)$ is the fraction of energy deposited into the grain, $L_{v}(v)$ is the luminosity of the source, $Q_{a b s}$ is the absorption coefficient for a given grain composition (a quantity which depends on the optical properties of the grains), and $r$ is the distance between the source and the dust grains. Regardless of the heating source, grains cool by emitting radiation with a rate given by

$$
L_{g r}(a)=4 \pi a^{2} \int \pi B_{v}(v, T) Q_{a b s}(v, a) d v,
$$

where $B_{v}(v, T)$ is the Planck blackbody function with $T$ as the grain temperature.

\section{Dust Formation in SNe}

Observations of high-redshift quasars at far-IR and sub-millimeter wavelengths revealed the presence of high masses of dust of $>10^{8} \mathrm{M}_{\odot}$, and implied that this dust had to form on relatively short timescales of a few hundred million years (see review by Gall et al. 2011). Since core-collapse SNe occur on short enough timescales, they were postulated to be the likely sources of dust in these young galaxies. Other possible mechanisms include the efficient growth of dust in the ISM (e.g. Draine 2009), and dust formation in outflowing quasar winds (Elvis et al. 2002). However, studies of extinction curves of high-redshift galaxies find that the flat shape of the curves in the optical and UV can be explained by dust grains processed by the SN reverse shock, providing further indication that $\mathrm{SNe}$ are the dominant contributors.

Dwek et al. (2009) used dust evolution models to fit the spectral energy distributions (SEDs) of high-redshift quasars, and determined that an average SN would need to produce $0.1-1.0 \mathrm{M}_{\odot}$ of dust to explain the observations, assuming that none of the grains are destroyed in the ISM. If dust destruction in the ISM is taken into account (see Section 6), the required mass would be even larger. For this reason, characterizing the masses and properties of dust grains produced in $\mathrm{SNe}$, as well as the efficiency of their eventual destruction in shocks, is critically important to understand the origin of dust in the early and present day universe.

While most of the non-stellar, baryonic mass in the ISM is hydrogen and helium, dust is comprised mostly of heavier metals, such as silicon, iron, oxygen, and carbon. These elements are found, in high abundances, in SN ejecta. Theoretical models for dust formation in $\mathrm{SNe}$ predict that significant masses of dust can form in these ejecta over the timescale of a few years post-explosion, but that the mass, composition, size, and ultimate survival of dust grains strongly depend on the type of the explosion and the metallicity (Kozasa et al. 2009). Models for dust formation in core-collapse SNe based on classical nucleation theory and the chemical kinematic approach for the formation of molecular precursors predict that 0.03-0.7 $M_{\odot}$ of dust can form in the ejecta, with some of the most abundant species being 
$\mathrm{MgSiO}_{3}, \mathrm{SiO}_{2}, \mathrm{Mg}_{2} \mathrm{SiO}_{4}$, and $\mathrm{C}$. However, only a fraction of this dust is expected to survive the SN reverse shock (see Section 6.2). The survival of dust grains is primarily determined by their size. Kozasa et al. (2009) found that Type IIb SNe with less massive envelopes, and therefore lower densities and higher velocities in the ejecta, tend to form smaller grains $(<0.006$ microns), while Type IIP SNe with massive envelopes form relatively large grain ( $>0.03$ microns). Sarangi \& Cherchneff (2015) explored dust formation in Type IIP SNe by accounting for the gas-phase chemistry, nucleation, and coagulation of grains, and confirmed the result that these explosions tend to form larger grains, especially if the ejecta are clumpy. They also found that clumpy ejecta lead to somewhat higher dust masses, and a higher fraction of metallic grains.

For SNe with a significant amount of pre-SN mass loss, it may be possible for dust to form in a cool, dense shell that forms in between the forward-shocked CSM and the reverse-shocked ejecta. Smith et al. (2007) report observations of the Type Ib SN 2006jc, which shows a rising near-IR continuum that is well-fit by hot graphite dust grains, in combination with progressively more asymmetrically blueshifted $\mathrm{He}$ I lines resulting from increasing dust obscuration of receeding material. Interestingly, these effects were seen at very early times, between 50 and 75 days, as opposed to the several hundreds of days necessary for dust to form in the ejecta.

Nozawa et al. (2011) modeled the formation of dust in Type Ia SNe and found that the total dust mass yield varies from $3 \times 10^{-4}$ to $0.2 M_{\odot}$, but that the lack of observational evidence for significant dust masses in Type Ia SNRs likely means that the formation of carbon grains is suppressed by energetic photons and electrons. They also find that the dust grains resulting from Type Ia explosions have sizes below 0.01 microns and that they will likely not survive the SN reverse shock. This implies that Type Ia SNe do not inject a significant amount of dust into the ISM.

\section{Observational Evidence for Ejecta Dust in SNe}

\subsection{SN 1987A}

Observations of SN 1987A in the Large Magellanic Cloud (LMC) provided the first direct evidence of dust condensation in SN ejecta and allowed monitoring of the evolution of the SN's IR emission as it transitioned to an SNR. The predictions that SN 1987A would form dust a few hundred days post-explosion (Gehrz \& Nye 1987) were confirmed when an IR excess was detected in the SN light curve (e.g. Moseley et al. 1989). The fraction of the SN bolometric luminosity reradiated by dust in the form of an IR continuum between 2-100 $\mu$ m evolved from $2 \%$ at day 260 postexplosion to as high as $83 \%$ at day 775 (Wooden et al. 1993). This coincided with the appearance of asymmetries in the optical emission lines, providing firm evidence that dust condensed in the ejecta of the $\mathrm{SN}$ explosion around day $\sim 500$. The early IR observations were consistent with $10^{-4}-4 \times 10^{-3} M_{\odot}$ of dust, radioactively 
Fig. 3 Top left: Hubble Space Telescope $\mathrm{H} \alpha$ line image of SN 1987A, showing the equatorial ring and the inner SN ejecta. Top right: ALMA $450 \mu \mathrm{m}$ continuum image, confirming that the dust emission at far-IR wavelengths spatially coincides with the SN ejecta. Bottom: IR photometric and spectroscopic data for SN 1987A. The additional cold dust component emitting in the $100-350 \mu \mathrm{m}$ range has been fit by $0.5 M_{\odot}$ of dust at $23 \mathrm{~K}$ (blue curve). Panels in the top row are from Indebetouw et al. (2014), and the bottom panel is from Matsuura et al. (2015).

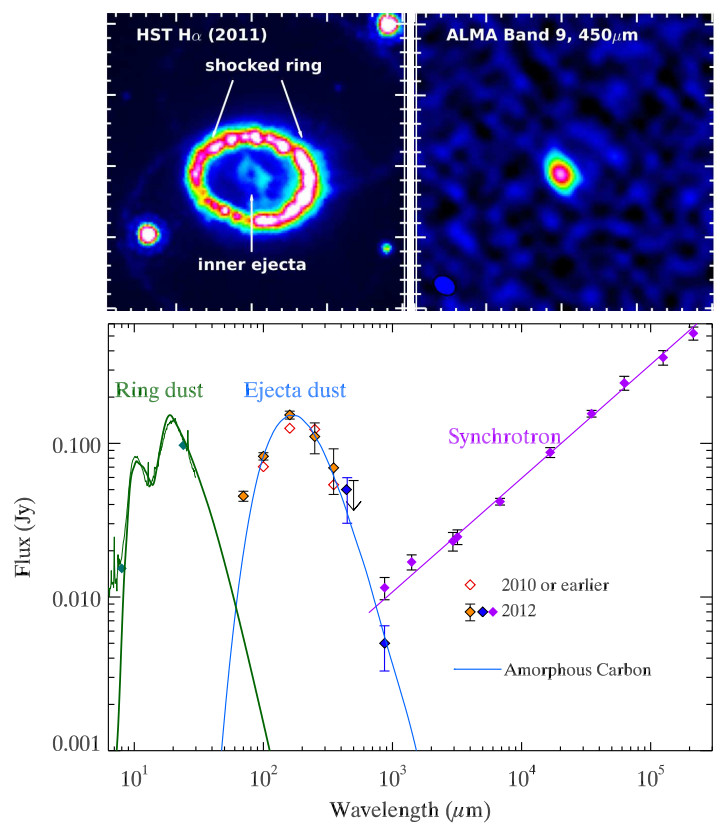

heated to a temperature of $\sim 100 \mathrm{~K}$, and consisting mostly of carbon grains, as inferred from the absence of silicate features in the IR spectrum (Wooden et al. 1993, Bouchet et al. 2004).

About ten years after explosion, as the blastwave of SN 1987A encountered the equatorial ring, the IR emission became completely dominated by pre-existing dust formed in the wind of the progenitor star. Mid-IR monitoring, particularly with Spitzer, revealed that the dust continuum emission could be explained by $\sim 10^{-5}-10^{-6} M_{\odot}$ of mostly silicate grains, collisionally heated by the X-ray emitting gas to a temperature of $\sim 180 \mathrm{~K}$ (e.g. Dwek et al. 2010). During this time, the mid-IR emission from the ejecta-condensed dust remained negligible.

An additional cold dust component in SN 1987A was discovered by Herschel in mid- and far-IR $(70-500 \mu \mathrm{m})$ observations. The component was attributed to $\sim 0.5$ $M_{\odot}$ of SN-formed dust, the largest mass ever detected in an SNR (Matsuura et al. 2015). The IR spectral energy distribution, shown in Figure 3 , was fit by $0.5 M_{\odot}$ of silicate and $0.3 M_{\odot}$ of carbon grains, emitting at a narrow temperature range of 20$25 \mathrm{~K}$. Curiously, these dust masses require a $100 \%$ condensation efficiency for $\mathrm{Si}$, and exceed the expected nucleosynthetic yields for carbon. High spatial resolution sub-millimeter observations with Atacama Large Millimeter/submillimeter Array (ALMA) confirmed that the dust emission originates from the central region of SN 1987A, coincident with the location of the SN ejecta (Indebetouw et al. 2014). The dominant heating source for the dust is likely the radioactive decay of ${ }^{44} \mathrm{Ti}$.

The fact that the currently observed mass of ejecta dust is orders of magnitude larger than the early estimates led several authors to suggest that the increase in mass 
was caused by gradual coagulation and accretion onto dust grains that formed at the early epochs, a few hundred days after the explosion (Ercolano et al. 2007, Wesson et al. 2015). Alternatively, Dwek et al. (2015) propose a scenario in which $\sim 0.4 M_{\odot}$ formed rapidly at the early epochs, but was hidden in optically thick regions of the ejecta. The total dust mass then evolves to $0.45 M_{\odot}$ at late times, consisting of $\sim$ $0.4 M_{\odot}$ of silicate and $0.05 M_{\odot}$ of carbon dust. While the proposed scenario is not unique, it alleviates the requirements for an unexpectedly large amount of $\mathrm{Si}, \mathrm{Mg}$, and $\mathrm{C}$ to be locked up in dust, and explains the absence of silicate emission features at the early epochs that would have been a result of self-absorption in the optically thick clumps. In either scenario, the large dust mass observed in SN 1987A is yet to encounter the SN reverse shock that is expected to destroy a significant fraction of the grains, so just how much of this dust will be injected into the ISM remains an open question.

\subsection{Extragalactic Observations}

Extragalactic (which we define here as beyond the Milky Way and any satellite galaxies, such as the MCs) observations in the IR are limited to the SN phase. Nonetheless, there have been several instances of dust being observed in extragalactic objects, both in emission, where the continuum from hot dust causes an excess above the normal SN light curve, and via indirect effects, such as asymmetric line profiles from dust obscuration of receding material.

One of the early results in this field came from Spitzer observations of SN 2003gd in NGC $628(9.3 \mathrm{Mpc})$ by Sugerman et al. (2006). The near-IR observations from 3.6 to $8 \mu \mathrm{m}$ clearly show a detection of the SN at day 499 that disappears by day 670. The authors concluded that as much as $0.02 M_{\odot}$ of dust had condensed in the ejecta within $\sim 1$ yr post-explosion. However, a later analysis by Meikle et al. (2007) came to the opposite conclusion, namely that the near and mid-IR emission from SN 2003gd could not be explained by newly formed dust, but instead was more consistent with a light echo from pre-existing dust.

Kotak et al. (2009) obtained Spitzer photometric and spectroscopic observations and optical spectra for several years post-explosion in the Type IIP SN 2004et. They fit their data with a three-component model which includes both emission from newly formed dust in the ejecta and an IR light echo from dust in the host galaxy. However, the amount of dust required to fit their observations was only about $10^{-4} M_{\odot}$, several orders of magnitude below the $\sim 0.1 M_{\odot}$ needed, per corecollapse SN, to reproduce the amount of dust in the universe. An extended analysis of this object out to beyond day 2000 by Fabbri et al. (2011) confirmed these results, without need for a large amount of dust formation.

Other observations of extragalactic SNe with Spitzer and other instruments produce similar results. Nozawa et al. (2008) used AKARI observations of the Type $\mathrm{Ib}$ SN 2006jc to derive a mass of only a few times $10^{-3} M_{\odot}$, despite theoretical calculations showing that about $1 M_{\odot}$ should form, based on the temperature evolution of 
the SN. The Type IIP SN 2004dj, quite close at only $3.5 \mathrm{Mpc}$, was observed several times with Spitzer between days 98 and 1381 post-explosion, but Szalai et al. (2011) find an upper limit of only $8 \times 10^{-4} M_{\odot}$. To date, no significant amount of mass has ever been seen in an extragalactic SN, though a caveat to this is that Spitzer may not be sensitive to a cold dust component, which could peak at wavelengths $>40$ $\mu \mathrm{m}$, or that the dust emitting region may be optically thick (see Section 4.1). While both Spitzer and Herschel had capabilities beyond this wavelength, their spatial resolution at long wavelengths made observations of SNe in other galaxies impossible. Once fully operational, ALMA may offer the possibility of detecting cold dust from distant $\mathrm{SNe}$ in the years following explosion.

\section{Observational Evidence for Ejecta Dust in SNRs}

\subsection{Ejecta Dust in Shell-Type SNRs}

Most of the evidence for dust in the ejecta of SNRs has come from the remnants of core-collapse $\mathrm{SNe}$, as would be expected from theoretical calculations (see Section 3). Perhaps the best example is Cas A, where Barlow et al. (2010) use Spitzer and Herschel observations to separate out the warm and cold dust components. The warm component arises from dust in the ISM heated by the forward shock, but the cold component, with a measured mass of $0.075 M_{\odot}$, is attributed to dust formed in the ejecta. However, this cold dust is located interior to and has not yet encountered the reverse shock, so the amount that will ultimately survive is still unknown (see Section 6). Arendt et al. (2014) decomposed the IR emission into several different dust components corresponding to various ejecta products in the remnant (see Figure 47).

Other core-collapse remnants have yielded similar results. Sandstrom et al. (2009) find only $\sim 10^{-3} M_{\odot}$ in the Small Magellanic Cloud remnant 1E0102-72.3. A survey of four LMC remnants by Williams et al. (2006) found $<0.1 M_{\odot}$ of dust in all of those objects, and they attribute most of that to swept-up ISM dust. They also point out that the morphology of the remnants in the IR more closely matches the forward-shocked emission seen in X-rays, rather than the SN ejecta.

Searches for dust in remnants of Type Ia SNe have turned up even smaller amounts of dust. Borkowski et al. (2006) examined several remnants in the LMC, and found no significant amounts of dust associated with the ejecta. Gomez et al. (2012b) report Herschel observations of Kepler's SNR and Tycho's SNR, and find no evidence for a cool component of dust associated with the ejecta in either remnant. 


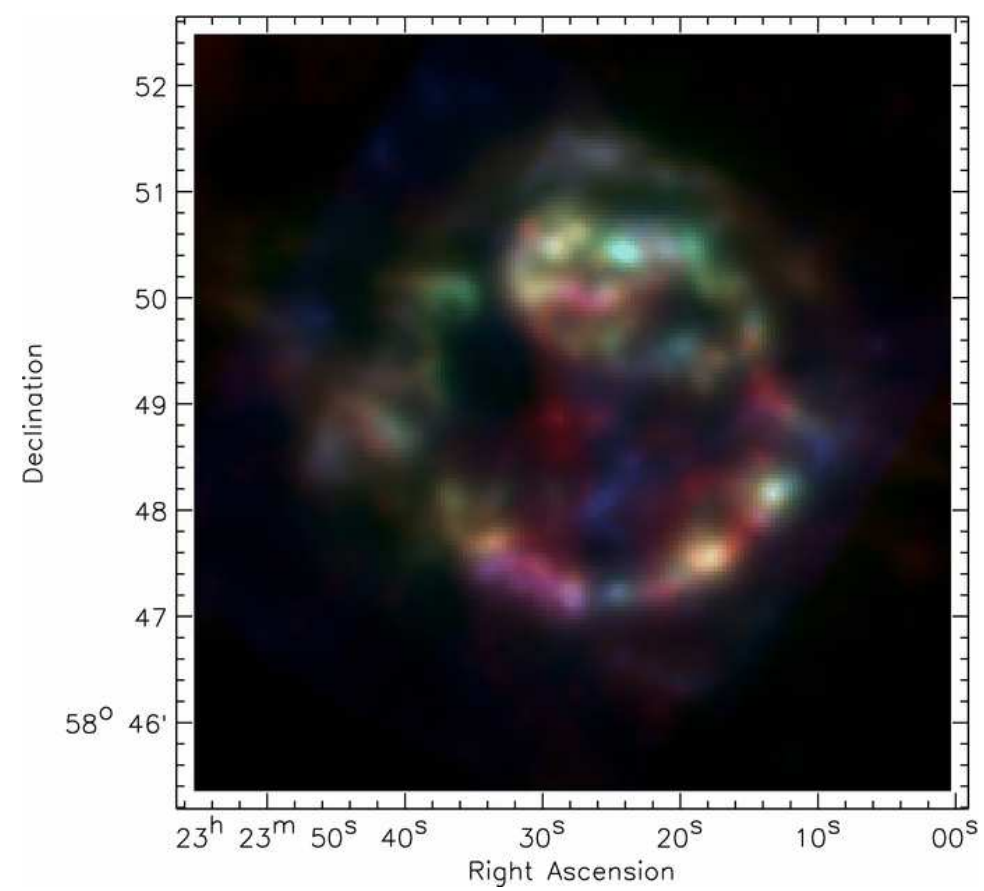

Fig. 4 A three-color image of Cas A (from Arendt et al. 2014), showing the dust continuum emission at $11.8 \mu \mathrm{m}$ in blue, $20.8 \mu \mathrm{m}$ in green, and $70 \mu \mathrm{m}$ in red. The various colors seen in the image reflect the spatial distribution of distinct species of dust that condensed in different layers of the SN ejecta.

\subsection{Ejecta Dust Around Pulsar Wind Nebulae}

Firm evidence for the existence of SN-formed dust is also found in SNRs that contain pulsar-generated wind nebulae. In the early stages of evolution of these systems, before the SN reverse shock has propagated towards the center of the SNR, the expanding pulsar wind nebula (PWN) drives a shock into the inner SN ejecta. Any dust that might have condensed in the ejecta can then be heated to IR-emitting temperatures, either collisionally by the shocked gas, or radiatively by the high-energy synchrotron emission from the PWN. The heating source provided by the central PWN therefore allows us to observe and study pristine SN dust before it has been processed by the $\mathrm{SN}$ reverse shock.

The most famous example of a system that exhibits this property is the Crab Nebula, whose PWN sweeps up SN ejecta material observed in the form of bright optical and IR filaments. The first observational evidence for dust in the Crab Nebula was found in the form of an IR excess above the synchrotron power-law in the integrated SED of the PWN, and attributed to $10^{-3}-10^{-2} M_{\odot}$ of carbon and/or 
silicate dust emitting at a temperature of $\sim 70 \mathrm{~K}$. Further evidence was found in the form of dust absorption features across the Crab's optical ejecta filaments.

The high values of the dust-to-gas mass ratio and the spatial correlation between the extinction features and low ionization emission lines in the filaments suggested that the dust in the Crab Nebula condensed from the SN ejecta, rather than originating from swept-up circumstellar material. Spitzer mid-IR spectroscopy confirmed that the dust continuum emission spatially correlates with the brightest filaments, and showed that the spectrum is mostly featureless (Temim et al. 2012b). The emission spectrum was fit by $(1.2-12) \times 10^{-3} M_{\odot}$ of warm dust, equally well described by either silicate or carbonaceous grains, radiating at a temperature of $55 \pm 4 \mathrm{~K}$ and $60 \pm 7 \mathrm{~K}$, respectively.

Far-IR Herschel observations of the Crab Nebula revealed the presence of a much more massive colder dust emission component $(\sim 30 \mathrm{~K})$ described by $0.24_{-0.08}^{+0.32} M_{\odot}$ of silicate or $0.11_{0.01}^{0.01} M_{\odot}$ of carbon dust (Gomez et al. 2012a). Temim \& Dwek (2013) modeled the mid and far-IR SED using a physical dust heating model, in which the broadband emission from the PWN radiatively heats dust grains with a continuous size distribution. They found that the resulting continuous temperature distribution of the grains leads to a reduction in the total dust mass $(0.019-0.13$ $M_{\odot}$ ), more in line with the expected nucleosynthetic yields for the Crab Nebula. On the other hand, Owen \& Barlow (2015) used a photoionization and radiative transfer model to show that the dust mass can be several times higher (up to $0.47 M_{\odot}$ ) if the grains reside in dense clumps. Both models suggest that the dust mass distribution is dominated by relatively large grains $(>0.1 \mu \mathrm{m})$, consistent with what is expected for a Type IIP SN.

Besides the Crab Nebula, three other PWNe show surrounding IR emission that likely originates from SN-formed dust; B0540-69.3 in the LMC (Williams et al. 2008), G54.1+0.3 (Temim et al. 2010), and Kes 75 (Temim et al. 2012a). Spitzer spectroscopy of B0540-69.3 shows a dust continuum excess above the PWN synchrotron emission that can be explained by a fairly small amount $\left(\sim 10^{-3} M_{\odot}\right)$ of dust, heated to a temperature of 50-65 K by the PWN shock (Williams et al. 2008). A multi-wavelength study of G54.1+0.3 by Temim et al. (2010) uncovered the unique nature of the IR shell surrounding the PWN. The highly broadened (up to $1000 \mathrm{~km} \mathrm{~s}^{-1}$ ) emission lines found in the Spitzer IRS spectrum (see Figure 5), which spatially correlate with the shell emission, confirmed that the shell is composed of SN ejecta and $\sim 0.1 M_{\odot}$ of newly-formed dust. Furthermore, the dust emission spectrum shows a distinct dust feature at $21 \mu \mathrm{m}$, produced by the same dust species as the $\mathrm{SN}$-condensed dust in Cas A. Even more interestingly, the dust is being heated not only by the PWN shock, but primarily by the $\mathrm{O}$ and B stars that are members of the cluster in which the SN progenitor existed. While the dust masses in these systems are relatively low, especially considering that the grains have not yet been encountered by the SN reverse shock that is expected to destroy a significant fraction, they were derived from Spitzer mid-IR observations that only sample the warmer grains. Far-IR emission has now been detected from all three systems with Herschel, suggesting that more dust resides at colder temperatures. Future studies and searches for IR emission around other young PWNe will be important for in- 


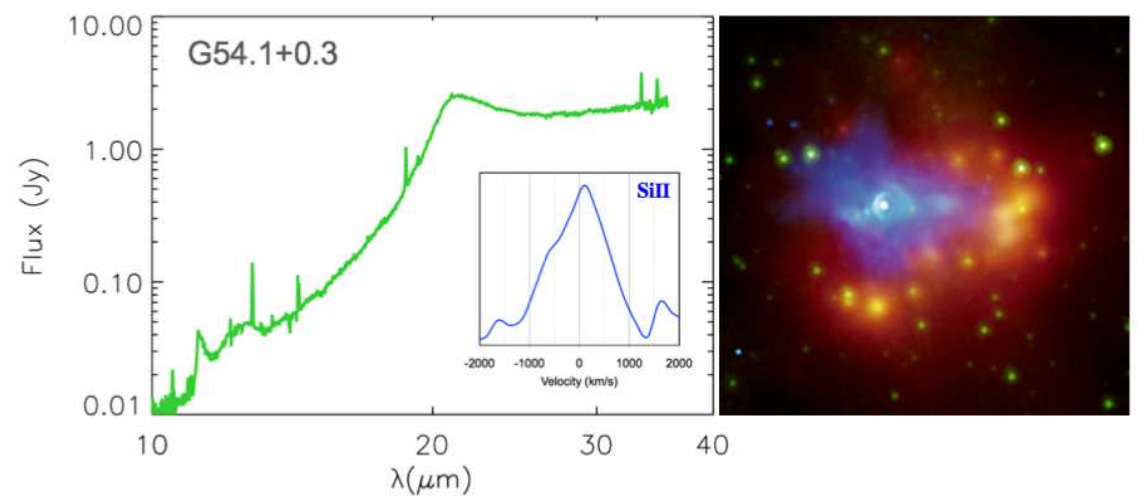

Fig. 5 Left: The Spitzer mid-IR spectrum of the shell surrounding the pulsar wind nebula in SNR G54.1+0.3. A zoomed in velocity profile of the [Si II] $34.8 \mu \mathrm{m}$ line is shown in blue in the inset. The dust spectrum shows mostly continuum emission, with a distinct broad feature peaking at $\sim$ $21 \mu \mathrm{m}$, arising from the same dust species as the ejecta dust in Cas A. The highly broadened emission line profiles, as shown by the Si line, indicate shell expansion velocities of $\sim 1000 \mathrm{~km} \mathrm{~s}^{-1}$ and confirm that the emission originates from SN ejecta. Right: A three-color composite image of SNR G54.1+0.3, showing the Chandra X-ray emission in blue, and Spitzer $8 \mu \mathrm{m}$ and $24 \mu \mathrm{m}$ emission in green and red, respectively. The pulsar wind nebula emitting in the X-rays is surrounded by IR emission from SN ejecta and dust. The dust is being heated both by the pulsar wind, and the surrounding stars, as it blows past them. The IR point sources that stand out in yellow arise from the hotter dust in the immediate vicinity of the stars. Image credit: X-ray: NASA/CXC/SAO/T.Temim et al. 2010; IR: NASA/JPL-Caltech

creasing the number of systems for which we can characterize the properties and masses of unprocessed SN dust.

\section{Dust Destruction in Shocks}

\subsection{Destruction Mechanisms}

Dust grains are generally destroyed via collisions, either with gas particles (sputtering) or with other grains (shattering). In sputtering, grains are bombarded by energetic protons and other ions. These collisions knock off a few atoms at a time, depending on the energy of the ion, gradually eroding the grain surface. To first order, the sputtering rate (i.e., the number of atoms knocked off per collision) is independent of the size of the grain, meaning that smaller grains are destroyed more quickly. This results in a modification of the shape of the grain size distribution with time. In Figure 6, we show an example of this, where the pre-shock and post-shock grain size distributions for both silicate and carbonaceous grains are shown after a processing time (years after the shock passage) of 1000 years. 
Fig. 6 The pre-shock (solid lines) and post-shock (dashed lines) dust grain size distributions of silicate and graphite dust grains 1000 years postshock. The density in this simulation was $\mathrm{n}_{0}=2 \mathrm{~cm}^{-3}$, with an ion temperature of 5 $\mathrm{keV}$. Silicate grains are shown in blue, while graphite is shown in green. Small grains are the most affected; see text for details. The vertical scales between silicate and graphite, reflecting the number of grains, are arbitrarily offset for ease of comparison, but the scales within each grain type are accurate.

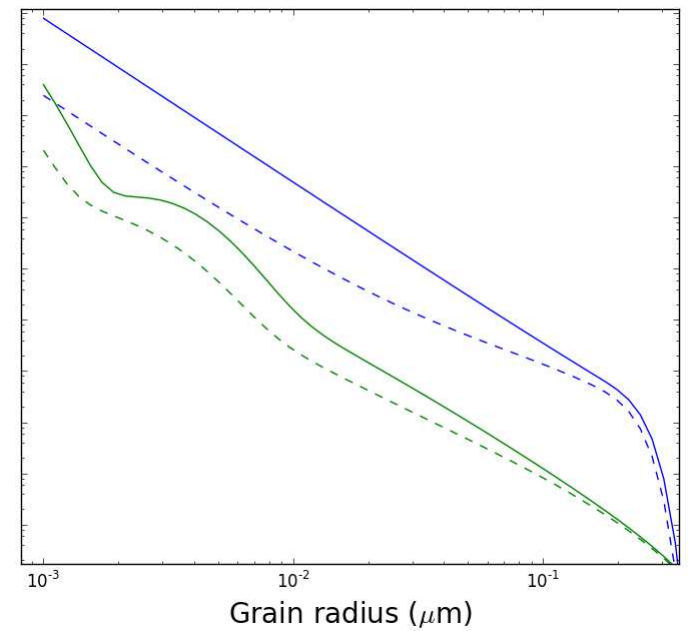

Thermal sputtering, described above, dominates the destruction of grains for fast, non-radiative shocks (Draine \& Salpeter 1979). For slower shocks, non-thermal sputtering, sometimes referred to as "ballistic" sputtering, is important as well. This sputtering arises from the relative gas-grain motions in the post-shock region. Dust grains generally have a low charge-to-mass ratio, and are largely unaffected by the passage of the shock (in SNRs, the shock wave is collisionless). The plasma around them, however, suddenly acquires a velocity of $(3 / 4) v_{s}$, where $v_{s}$ is the shock speed. They are eventually brought to rest in the frame of the gas due to the drag force from collisions with ions, but this takes a non-negligible amount of time, during which their sputtering rate is increased.

Finally, shattering of grains via grain-grain collisions reprocesses grains by reducing their size. While not a destruction mechanism, per se, since the mass remains in the solid dust phase, this process also affects the grain size distribution, tilting it towards smaller grains that are then more easily destroyed by sputtering. Jones et al. (1996) show that for slow shocks of $<200 \mathrm{~km} \mathrm{~s}^{-1}, 5-15 \%$ of the initial mass in grains may be reprocessed via shattering to very small grain sizes of less than $1 \mathrm{~nm}$, and thus may be a significant source of polycyclic aromatic hydrocarbons (PAHs) and other small grains.

\subsection{Destruction of SN Dust by the Reverse Shock}

While core-collapse $\mathrm{SNe}$ are capable of producing a significant amount of dust from their ejecta, the ultimate survival of this dust following the passage of the SN reverse shock determines whether they are indeed major sources of dust in galaxies. The survival of dust grains depends not only on the type of explosion (see Section 3.3), but 
also on the clumpiness of SN ejecta, and the density structure of the surrounding ISM. The theoretical models generally show that $40-100 \%$ of the dust mass is destroyed in the encounter with the reverse shock, and that grains with sizes below 0.1 $\mu \mathrm{m}$ are completely sputtered away (e.g. Kozasa et al. 2009, Silvia et al. 2010).

While the dust produced in Type Ia and IIb SNe is shown to be almost completely destroyed, even for the highest gas densities and clumpy ejecta, as much as $0.2 \mathrm{M}_{\odot}$ may survive in Type IIP SNRs due to the larger size of grains that condense in the ejecta (Kozasa et al. 2009, Nozawa et al. 2011, Biscaro \& Cherchneff 2014). We note that for all SNRs in which significant dust masses have been discovered to date (SN 1987A, Cas A, Crab Nebula, and G54.1+0.3), the dust resides on the inside of the reverse shock and is yet to be processed. So far, the only observational evidence for $\mathrm{SN}$ dust surviving the reverse shock is the discovery of $0.02 \mathrm{M}_{\odot}$ of dust in the interior of a 10,000-year-old SNR Sgr A East (Lau et al. 2015).

\subsection{Observational Evidence for Destruction in SNRs}

Modeling the IR emission from individual remnants shows direct evidence for the destruction of ISM dust via sputtering. Borkowski et al. (2006) and Williams et al. (2006) used early Spitzer imaging observations of young SNRs in the LMC to show that the observed fluxes could not be modeled by standard, unmodified grain size distributions. The fits required dust destruction of order $\sim 25-50 \%$ of the mass in grains encountered by the forward shock. Blair et al. (2007) did the same for Kepler's SNR, where the high densities encountered by the forward shock lead to $\sim$ $75 \%$ of the grains being destroyed. Arendt et al. (2010) observed Puppis A, an older galactic remnant interacting with a molecular cloud, to reach a similar conclusion. Since Puppis A is a large remnant, they were able to place the Spitzer spectroscopic slits at various points behind the shock and follow the evolution of the dust grain size distribution. They found that the Spitzer spectra could only be fit by removing 25\% of the mass in grains. A similar conclusion was reached by Sankrit et al. (2014) in observations of the Cygnus Loop, another older remnant occupying a large volume of the ISM. It is important to note that grain destruction does not merely lower the IR flux from the remnant, but also changes the shape of the spectrum, due to small grains being preferentially destroyed.

Spitzer is now in its warm phase and no longer has spectroscopic capabilities. However, the James Webb Space Telescope (JWST) is scheduled for launch in 2018 , and will have both imaging and spectroscopic capabilities from $\sim 1-25 \mu \mathrm{m}$. JWST's angular resolution will be nearly an order of magnitude better than Spitzer's, and will allow studies like the one done on Puppis A to be done on several remnants, both in the Galaxy and the MCs. The much greater sensitivity means that fainter remnants can be studied as well. 


\subsection{Supernovae as Net Destroyers of Dust?}

Besides potentially being important sources of dust in galaxies, SNe are also responsible for the majority of dust destruction in the ISM. The efficiency of the destruction depends on the ISM conditions, such as the gas density, homogeneity, metallicity, magnetic field, and dust-to-gas mass ratio, as well as on the properties of the ISM dust grains themselves. Various theoretical models have been developed to study the effect of SN blastwaves on dust in the ISM, and they generally agree that SN dust destruction rates exceed the SN injection rates by at least an order of magnitude (Jones et al. 1996). Slavin et al. (2015) modeled dust destruction by radiative SNRs expanding in a homogeneous, magnetized, warm phase of the ISM, and found that a typical SNR in the Galaxy would be expected to destroy $\sim 3.7 \mathrm{M}_{\odot}$ of silicate and $\sim 1.4 \mathrm{M}_{\odot}$ of carbon dust, significantly more than a typical $\mathrm{SN}$ is expected to produce (see Section 3). This discrepancy implies that dust in the ISM is either shielded somehow, or that a large fraction grows in dense molecular clouds.

In a recent study, Temim et al. (2015) estimated the global dust destruction rate by a complete sample of SNRs in the MCs by combining the observational constraints on the dust-to-gas mass ratio and gas density around each SNR, and the theoretically determined dust destruction efficiencies. They calculated the total amount of dust each SNR will destroy throughout its evolution, and found that on average, $7.7 \pm 2.7 \mathrm{M}_{\odot}$ is destroyed by an SNR in the LMC and $3.3 \pm 1.8 \mathrm{M}_{\odot}$ in the SMC. Lakicevic et al. (2015) estimated the amount of dust destruction in the LMC by comparing the dust mass surface densities inside and outside the SNRs with their surrounding media. They found evidence for dust destruction in six SNRs, with an average of $6.5 \mathrm{M}_{\odot}$ destroyed. Ochsendorf et al. (2015) find that dust processing by SNRs inside superbubbles in the LMC increases the dust destruction efficiency even further, since dust in continually replenished through cloud evaporations inside the bubbles and destroyed by subsequent SNe. These studies support the idea that SNe are net destroyers of dust in the ISM, at least in present-day galaxies.

\section{Conclusions}

In light of recent IR observations of young SNRs, particularly SN 1987A, there is no doubt that $\mathrm{SNe}$ can form significant quantities of dust. However, the properties and amount of dust produced in different progenitor types, as well as the fraction of dust that ultimately survives the remnant phase to be injected into the ISM, are not well known. Additionally, since the SNR shock waves destroy significant quantities of ISM dust, it is likely that SNe, as a whole, are net destroyers of dust in galaxies. Significant progress in this field will be made upon the successful launch of JWST, a mid-IR observatory with a resolution about an order of magnitude better than Spitzer and sensitivity several hundred times higher. JWST will allow the followup of extragalactic SNe for tens of years in some cases, and will serve as a valuable monitor on SN 1987As continuing evolution. The superior resolution will also fur- 
ther disentangle the various dust components in SNRs in both the Galaxy and the MCs, and allow studies of the efficiency of dust destruction by SNR shocks. A combination of observations of both SNe and SNRs, along with theoretical modeling, will be necessary to solve the mystery of the origin of dust in the universe.

\section{References}

1. R.G. Arendt, E. Dwek, W.P. Blair, P. Ghavamian, U. Hwang, K.S. Long, et al. 2010, Spitzer Observations of Dust Destruction in the Puppis A Supernova Remnant, ApJ, 725, 585

2. R.G. Arendt, E. Dwek, G. Kober, J. Rho, \& U. Hwang 2014, Interstellar and Ejecta Dust in the Cas A Supernova Remnant, ApJ, 786, 55

3. M.J. Barlow, O. Krause, B.M. Swinyard, B. Sibthorpe, M.-A. Besel, R. Wesson, et al. 2010, A Herschel PACS and SPIRE study of the dust content of the Cassiopeia A supernova remnant, A\&A, 518, 138

4. R. Becker \& W. Doring 1935, Kínetische Behandlung der Keimbildung in übersättigten Dämpfen, Annalen der Physik, 24, 719

5. C. Biscaro, \& I. Cherchneff, 2014, Molecules and dust in Cassiopeia A. I. Synthesis in the supernova phase and processing by the reverse shock in the clumpy remnant, A\&A, 564, A25

6. W.P. Blair, P. Ghavamian, K.S. Long, B.J. Williams, K.J. Borkowski, S.P. Reynolds, et al. 2007, Spitzer Space Telescope Observations of Kepler's Supernova Remnant: A Detailed Look at the Circumstellar Dust Component, ApJ, 662,998

7. K.J. Borkowski, B.J. Williams, S.P. Reynolds, W.P. Blair, P. Ghavamian, R. Sankrit, et al. 2006, Dust Destruction in Type Ia Supernova Remnants in the Large Magellanic Cloud, ApJ, 642, 141

8. P. Bouchet, J.M. De Buizer, N.B. Suntzeff, J.I. Danziger, T.L.Hayward, C.M. Telesco, et al. 2004, High-Resolution Mid-infrared Imaging of SN 1987A, ApJ, 611, 394

9. B.T. Draine \& Salpeter, E.E., 1979, Destruction mechanisms for interstellar dust, ApJ, 231, 77

10. B. T. Draine, 2009, Cosmic Dust - Near and Far ASP Conference Series, Vol. 414, proceedings of a conference held 8-12 September 2008 in Heidelberg, Germany. Edited by Thomas Henning, Eberhard Grn, and Jrgen Steinacker. San Francisco: Astromomical Society of the Pacific, 414, 453

11. E. Dwek, F. Galliano, \& A. Jones, 2009, Cosmic Dust - Near and Far ASP Conference Series, Vol. 414, proceedings of a conference held 8-12 September 2008 in Heidelberg, Germany. Edited by Thomas Henning, Eberhard Grn, and Jrgen Steinacker. San Francisco: Astromomical Society of the Pacific, 414, 183

12. E. Dwek, R.G. Arendt, P. Bouchet, D.N. Burrows, P. Challis, J.I. Danziger, et al. 2010, Five Years of Mid-infrared Evolution of the Remnant of SN 1987A: The Encounter Between the Blast Wave and the Dusty Equatorial Ring, ApJ, 722, 425

13. M. Elvis, M. Marengo, \& M. Karovska, 2002, Smoking Quasars: A New Source for Cosmic Dust, ApJL, 567, L107

14. B. Ercolano, M. J. Barlow, \& B. E. K. Sugerman, 2007, Dust yields in clumpy supernova shells: SN 1987A revisited, MNRAS, 375, 753

15. J. Fabbri, M. Otsuka, M.J. Barlow, J.S. Gallagher, R. Wesson, B.E.K. Sugerman, et al. 2011, The effects of dust on the optical and infrared evolution of SN 2004et, MNRAS, 418, 1285

16. C. Gall, J. Hjorth, \& A. C. Andersen, 2011, Production of dust by massive stars at high redshift, A\&ARv, 19, 43

17. R. D. Gehrz,\& E. P. Ney, 1987, On the Possibility of Dust Condensation in the Ejecta of Supernova 1987a, Proceedings of the National Academy of Science, 84, 6961

18. H. L. Gomez, O. Krause, M.J. Barlow, B.M. Swinyard, P.J. Owen, C.J.R. Clark, et al. 2012a, A Cool Dust Factory in the Crab Nebula: A Herschel Study of the Filaments, ApJ, 760, 96 
19. H.L. Gomez, C.J.R. Clark, T. Nozawa, O. Krause, E.L. Gomez, M. Matsuura, et al. 2012b, Dust in historical Galactic Type Ia supernova remnants with Herschel, MNRAS, 420, 3557

20. R. Indebetouw, M. Matsuura, E. Dwek, G. Zanardo, M.J. Barlow, M. Baes, et al. 2014, Dust Production and Particle Acceleration in Supernova 1987A Revealed with ALMA, ApJL, 782, L2

21. A. P. Jones, A. G. G. M. Tielens, \& D. J. Hollenbach, 1996, Grain Shattering in Shocks: The Interstellar Grain Size Distribution, ApJ, 469, 740

22. R. Kotak, W.P.S. Meikle, D. Farrah, C.L. Gerardy, R.J. Foley, S.D. Van Dyk, et al. 2009, Dust and The Type II-Plateau Supernova 2004et, ApJ, 704, 306

23. T. Kozasa, T. Nozawa, N. Tominaga, H. Umeda, K. Maeda, K. Nomoto 2009, Cosmic Dust - Near and Far ASP Conference Series, Vol. 414, proceedings of a conference held 8-12 September 2008 in Heidelberg, Germany. Edited by Thomas Henning, Eberhard Grn, and Jrgen Steinacker. San Francisco: Astromomical Society of the Pacific, 414, 43

24. R. M. Lau, T. L. Herter, M. R. Morris, Z. Li, \& J. D. Adams, 2015, Old supernova dust factory revealed at the Galactic center, Science, 348, 413

25. M. Matsuura, E. Dwek, M.J. Barlow, B. Babler, M. Baes, M. Meixner, et al. 2015, A Stubbornly Large Mass of Cold Dust in the Ejecta of Supernova 1987A, ApJ, 800, 50

26. W.P.S. Meikle, S. Mattila, A. Pastorello, C.L. Gerardy, R. Kotak, J. Sollerman, et al. 2007, A Spitzer Space Telescope Study of SN 2003gd: Still No Direct Evidence that Core-Collapse Supernovae are Major Dust Factories, ApJ, 665, 608

27. T. Nozawa, T. Kozasa, N. Tominaga, I. Sakon, M. Tanaka, T. Suzuki, et al. 2008, Early Formation of Dust in the Ejecta of Type Ib SN 2006jc and Temperature and Mass of the Dust, ApJ, 684, 1343

28. T. Nozawa, K. Maeda, T. Kozasa, M. Tanaka, K. Nomoto, \& H. Umeda, 2011, Formation of Dust in the Ejecta of Type Ia Supernovae, ApJ, 736, 45

29. B. B. Ochsendorf, A. G. A. Brown, J. Bally, \& A. G. G. M. Tielens, 2015, Nested Shells Reveal the Rejuvenation of the Orion-Eridanus Superbubble, ApJ, 808, 111

30. P. J. Owen, \& M. J. Barlow 2015, The Dust and Gas Content of the Crab Nebula, ApJ, 801, 141

31. W.T. Reach, J. Rho, A. Tappe, T.G. Pannuti, C.L. Brogan, E.B. Churchwell, et al. 2006, A Spitzer Space Telescope Infrared Survey of Supernova Remnants in the Inner Galaxy, AJ, 131, 1479

32. K.M. Sandstrom, A.D. Bolatto, D. Alberto, S. Stanimirović, J. van Loon, J.D.T. Smith, 2009, Measuring Dust Production in the Small Magellanic Cloud Core-Collapse Supernova Remnant 1E 0102.2-7219, ApJ, 696, 2138

33. R. Sankrit, J.C. Raymond, M. Bautista, T.J. Gaetz, B.J. Williams, W.P. Blair, et al. 2014, Spitzer IRS Observations of the XA Region in the Cygnus Loop Supernova Remnant, ApJ, 787, 3

34. A. Sarangi, \& I. Cherchneff, 2015, Condensation of dust in the ejecta of Type II-P supernovae, A\&A, 575, A95

35. D. W. Silvia, B. D. Smith, \& J. M. Shull, 2010, Numerical Simulations of Supernova Dust Destruction. I. Cloud-crushing and Post-processed Grain Sputtering, ApJ, 715, 1575

36. J. D. Slavin, E. Dwek, \& A. P. Jones, 2015, Destruction of Interstellar Dust in Evolving Supernova Remnant Shock Waves, ApJ, 803, 7

37. N. Smith, R.J. Foley, A.V. Filippenko, 2008, Dust Formation and He II 4686 Emission in the Dense Shell of the Peculiar Type Ib Supernova 2006jc, ApJ, 680, 568

38. B.E. Sugerman, B. Ercolano, M.J. Barlow, A.G.G.M. Tielens, G.C. Clayton, A.A. Zijlstra, et al. 2006, Massive-Star Supernovae as Major Dust Factories, Science, 313, 196

39. T. Szalai, J. Vinkó, Z. Balog, A. Gáspár, M. Block, L.L. Kiss, 2011, Dust formation in the ejecta of the type II-P supernova 2004dj, A\&A, 527, 61

40. T. Temim, P. Slane, S. P. Reynolds, J. C. Raymond, \& K. J. Borkowski, 2010, Deep Chandra Observations of the Crab-like Pulsar Wind Nebula G54.1+0.3 and Spitzer Spectroscopy of the Associated Infrared Shell, ApJ, 710, 309 
41. T. Temim, P. Slane, R. G. Arendt, \& E. Dwek, 2012a, Infrared and X-Ray Spectroscopy of the Kes 75 Supernova Remnant Shell: Characterizing the Dust and Gas Properties, ApJ, 745, 46

42. T. Temim, G. Sonneborn, E. Dwek, R.G. Arendt, R.D. Gehrz, P. Slane, et al. 2012b, Properties and Spatial Distribution of Dust Emission in the Crab Nebula, ApJ, 753, 72

43. T. Temim, \& E. Dwek, 2013, The Importance of Physical Models for Deriving Dust Masses and Grain Size Distributions in Supernova Ejecta. I. Radiatively Heated Dust in the Crab Nebula, ApJ, 774, 8

44. T. Temim, E. Dwek, K. Tchernyshyov, M.L. Boyer, M. Meixner, C. Gall, et al. 2015, Dust Destruction Rates and Lifetimes in the Magellanic Clouds, ApJ, 799, 158

45. R. Wesson, R., M. J. Barlow, M. Matsuura, \& B. Ercolano, 2015, The timing and location of dust formation in the remnant of SN 1987A, MNRAS, 446, 2089

46. B.J. Williams, K.J. Borkowski, S.P. Reynolds, W.P. Blair, P. Ghavamian, S.P. Hendrick, et al., 2006, Dust Destruction in Fast Shocks of Core-Collapse Supernova Remnants in the Large Magellanic Cloud, ApJ, 652, 33

47. B.J. Williams, K.J. Borkowski, S.P. Reynolds, J.C. Raymond, K.S. Long, J. Morse, et al., 2008, Ejecta, Dust, and Synchrotron Radiation in SNR B0540-69.3: A More Crab-Like Remnant than the Crab, ApJ, 687, 1054

48. B.J. Williams, K.J. Borkowski, S.P. Reynolds, P. Ghavamian, J.C. Raymond, K.S. Long, et al., 2011, Dusty Blast Waves of Two Young Large Magellanic Cloud Supernova Remnants: Constraints on Post-shock Compression, ApJ, 729, 65

49. B.J. Williams, K.J. Borkowski, S.P. Reynolds, P. Ghavamian, W.P. Blair, K.S. Long, et al. 2012, Dust in a Type Ia Supernova Progenitor: Spitzer Spectroscopy of Kepler's Supernova Remnant, ApJ, 755, 3

50. D. H. Wooden, D.M. Rank, J.D. Bregman, F.C. Witteborn, A.G.G.M. Tielens, M. Cohen, et al. 1993, Airborne spectrophotometry of SN 1987A from 1.7 to 12.6 microns - Time history of the dust continuum and line emission, ApJS, 88, 477

152, 211 